Nonlin. Processes Geophys., 20, 1-10, 2013

www.nonlin-processes-geophys.net/20/1/2013/

doi:10.5194/npg-20-1-2013

(C) Author(s) 2013. CC Attribution 3.0 License.

\title{
Dynamics of a two-fault system with viscoelastic coupling
}

\author{
A. Amendola and M. Dragoni \\ Dipartimento di Fisica, Università di Bologna, Viale Carlo Berti Pichat 8, 40127 Bologna, Italy \\ Correspondence to: A. Amendola (alfonso.amendola2@unibo.it)
}

Received: 7 September 2012 - Revised: 28 November 2012 - Accepted: 29 November 2012 - Published: 4 January 2013

\begin{abstract}
A fault system made of two segments or asperities subject to a constant strain rate is considered. The fault is modelled as a discrete dynamical system made of two blocks coupled by a Maxwell spring dashpot element and pulled at constant velocity on a rough plane. The long-term behaviour of the fault is studied by calculating the orbits of the system in the phase space. The model shows the role of viscoelastic relaxation in the Earth's crust in controlling the occurrence times of earthquakes. If a viscoelastic coupling is present, earthquakes are anticipated or delayed with respect to the elastic case. The limit cycles made of two alternate asperity failures, which are observed in the case of purely elastic coupling, are no longer produced. The model is applied to the 1964 Alaska earthquake, which was the effect of the failure of two asperities and for which a remarkable post-seismic relaxation has been observed in the subsequent decades. In such a fault system, viscoelastic coupling of the asperities appears to have a great influence on the occurrence times of earthquakes.
\end{abstract}

\section{Introduction}

The seismic activity of a fault is controlled by the applied tectonic stress, due to plate motion, and by the stresses transferred by dislocations on neighbouring faults. Therefore fault interaction plays a key role in determining the occurrence times of earthquakes. Many studies have been made on this subject and reviews can be found in Harris (1998), Stein (1999) and Steacy et al. (2005).

A role in this process is also played by the rheological properties of the Earth's crust. Crustal rocks are not perfectly elastic, but have a certain degree of anelasticity (Carter, 1976; Kirby, 1983; Kirby and Kronenberg, 1987; Ranalli, 1995; Nishimura and Thatcher, 2003; Bürgmann and Dresen, 2008). As a consequence, the static stress fields produced by seismic dislocations undergo a certain amount of relaxation during the interseismic intervals, which may have durations of hundreds of years (Chen and Molnar, 1983; Dragoni et al., 1986; Kusznir, 1991; Kenner and Segall, 2000). In a fault system, such a long-term variation of stress alters the stress distribution on faults and modifies the occurrence times of seismic events (e.g. Chéry et al., 2001; Lynch et al., 2003; Smith and Sandwell, 2006; Piombo et al., 2007).

In addition to bulk relaxation of the crust, creep on fault segments may take place (e.g. Marone et al., 1991; Belardinelli and Bonafede, 1995; Heki et al., 1997). The interaction between two faults due to aseismic slip was studied by Dragoni and Tallarico (1992) and Tallarico et al. (2002) in the framework of continuum mechanics.

In the present paper, we study a two-fault system where the stress transferred from a fault to another one partially relaxes during an interseismic interval. To this aim we consider a discrete dynamical system, made of two blocks pulled by a driver at constant velocity on a rough plane. The coupling between the blocks is realised by a spring and a dashpot in series: this amounts to assume a Maxwell viscoelastic rheology for the Earth's crust. We do not consider aseismic slip on faults in the present paper.

Discrete dynamical systems are usefully employed as models to investigate the long-term behaviour of faults. Such models include the essential features of seismic sources, i.e. a stick-slip mechanism activated by applied forces, but avoid the detailed field description of continuum mechanics. The advantage is that we can follow the evolution of the system in the phase space and obtain a deeper understanding of the long-term behaviour of the system itself.

In the case of a purely elastic coupling, the two-block model has been proposed by Nussbaum and Ruina (1987) and further investigated by Huang and Turcotte (1990a, b, 1992), McCloskey and Bean (1992), de Sousa Vieira (1995), Turcotte (1997), He (2003), Dragoni and Santini (2010, 


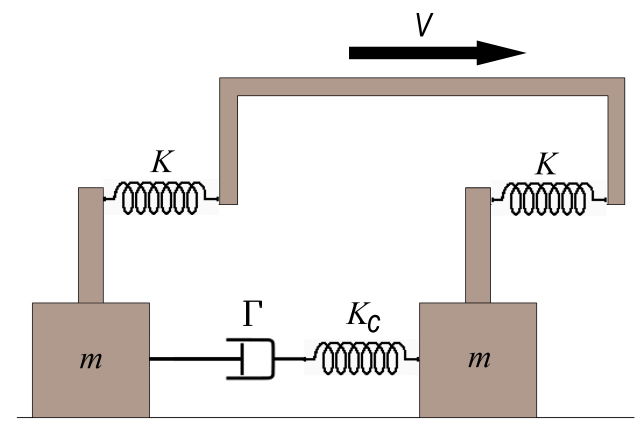

Fig. 1. Sketch of the model.

2011). In the case of uniform friction, the long-term behaviour of the system in the phase space is one of an infinite number of limit cycles producing an alternate motion of the blocks, representing an alternate slip of the faults. We shall show how this picture is modified by the introduction of viscoelastic coupling.

Studies of block systems with viscoelastic coupling have been already presented by some authors. A review of such work up to year 2000 was given by Pelletier (2000). In particular Hainzl at al. (1999) considered a network of interconnected blocks lying between two tectonic plates in the form of a continuous cellular automaton. Kenner and $\mathrm{Si}$ mons (2005) considered a spring-dashpot-slider system simulating the stress interactions that take place in a three-layer model composed of an elastic plate overlying a viscoelastic channel above a viscoelastic half-space.

\section{The model}

We consider a system made of two blocks (named 1 and 2) having equal mass $m$ and placed on a horizontal plane (Fig. 1). Each block is connected by a horizontal spring to a driving mechanism moving at constant velocity $v$ in the horizontal direction. The two springs (named 1 and 2) have the same rigidity $K$. The blocks are connected to each other by spring 3 with rigidity $K_{c}$ in series with a dashpot with damping constant $\Gamma$. The Maxwell relaxation time is

$\tau=\frac{\Gamma}{K_{c}}$.

The Maxwell body is the simplest viscoelastic model that is representative of the long-term behaviour of crustal rocks and is considered to be appropriate in the framework of the model assumptions. An analogous viscoelastic coupling is not introduced for the driving mechanism, since we assume that the characteristic Maxwell time associated with tectonic loading is much larger than typical interseismic intervals; therefore, the behaviour can be approximated as elastic. If this assumption is not made, the stress imposed to the faults by plate motion would approach a constant value and earthquakes would no longer occur. We assume that the motion of each block is resisted by a static friction $f_{\mathrm{s}}$ and a dynamic friction $f_{\mathrm{d}}$.

The state of the system can be described by three variables $x, y$ and $z$ measuring the extensions of springs 1,2 and 3 , respectively, as functions of time $t$. Accordingly, the system has three degrees of freedom and the phase space is a 6-manifold. We introduce non-dimensional variables

$X=\frac{K x}{f_{\mathrm{s}}}, \quad Y=\frac{K y}{f_{\mathrm{s}}}, \quad Z=\frac{K z}{f_{\mathrm{s}}}, \quad T=\sqrt{\frac{K}{m}} t$.

The system is described by four non-dimensional parameters that are defined as follows:

$\alpha=\frac{K_{c}}{K}, \quad \epsilon=\frac{f_{\mathrm{d}}}{f_{\mathrm{s}}}, \quad V=\frac{\sqrt{K m}}{f_{\mathrm{s}}} v, \quad \Theta=\sqrt{\frac{K}{m}} \tau$.

If $f_{1}$ and $f_{2}$ are the forces applied to blocks 1 and 2, respectively, we define non-dimensional forces

$F_{1}=\frac{f_{1}}{f_{\mathrm{s}}}, \quad F_{2}=\frac{f_{2}}{f_{\mathrm{s}}}$.

They can be written in terms of the model variables as

$F_{1}=-X+\alpha Z, \quad F_{2}=-Y-\alpha Z$

which reduce to the expressions for the elastic case if $Z=$ $Y-X$.

In view of the seismological application, the system is subject to a number of constraints. The driver velocity $V$ is constant and positive. The degree of coupling $\alpha$ is a real number greater or equal to zero. The ratio $\epsilon$ is a positive real number smaller than 1 .

Since the sign of shear stress on a fault does not change in time, springs 1 and 2 are always stretched or at rest, so that $X \geq 0, Y \geq 0$. In addition $X$ and $Y$ can not exceed 1 (corresponding to static friction), while the maximum value of $Z$ is $Y-X$. Then

$0 \leq X \leq 1, \quad 0 \leq Y \leq 1, \quad-1 \leq Z \leq 1$.

Therefore, the projection of the phase space in the space $X Y Z$ is the parallelepiped defined by Eq. (6). The conditions on $X$ and $Y$ entail an additional condition on $\epsilon$ : since the maximum value of slip is $2(1-\epsilon)$, which takes place when $\alpha=0, X$ and $Y$ satisfy the condition of being greater or equal to zero only if $2(1-\epsilon) \leq 1$ or $\epsilon \geq 1 / 2$.

The dynamics of the system has four different modes, corresponding to stationary blocks (mode 00$)$, motion of block 1 (mode 10), motion of block 2 (mode 01 ), simultaneous motion of blocks 1 and 2 (mode 11). Each mode is described by a different system of autonomous differential equations. Therefore, the dynamical system is piecewise smooth and has a discontinuous right hand side: it is a Filippov system (Filippov, 1988; di Bernardo et al., 2008).

The conditions for the onset of motion of blocks 1 and 2 are respectively

$F_{1}=-1, \quad F_{2}=-1$

or 


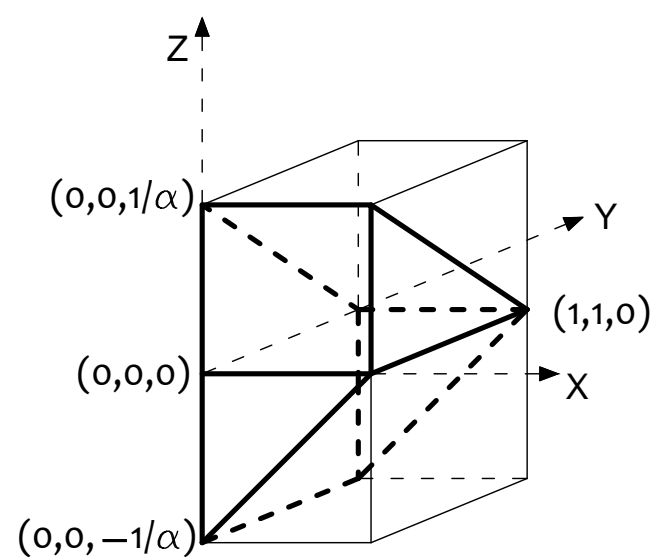

Fig. 2. The sticking region $H$.

$X-\alpha Z-1=0, \quad Y+\alpha Z-1=0$.

These are the equations of two planes that we call plane 1 and plane 2 , respectively. They have in common the point $(1,1,0)$.

In mode 00 , the velocities $\dot{X}, \dot{Y}$ and $\dot{Z}$ can be neglected. The region of phase space including the states in which the blocks are stationary (sticking region) is therefore a subset of the hyperplane $\dot{X}=0, \dot{Y}=0, \dot{Z}=0$. It is defined as the set of solutions of the six linear disequations

$0 \leq X \leq 1, \quad 0 \leq Y \leq 1, \quad \frac{X-1}{\alpha} \leq Z \leq \frac{1-Y}{\alpha}$.

It is therefore a convex hexahedron $H$ (Fig. 2). Its faces are 2 trapezoids, 2 rectangles and 2 triangles and its volume is equal to $1 / \alpha$. The hexahedron is divided in half by the plane $Z=0$ and its section is a square of unit side on this plane. The projections of the orbits of the system on the plane $Z=0$ are contained in this square.

\section{Solutions}

We now write down the equations of motions of the four modes and give the corresponding solutions.

\subsection{Stationary blocks (mode 00)}

The variables $X$ and $Y$ obey the same equations as in the case of elastic coupling, while $Z$ is governed by the Maxwell constitutive equation. The equations of motion are then

$$
\ddot{X}=0, \quad \ddot{Y}=0, \quad \ddot{Z}=\frac{Z}{\Theta^{2}}
$$

where a dot indicates differentiation with respect to $T$. With initial conditions

$$
\begin{array}{lll}
X(0)=\bar{X}, & Y(0)=\bar{Y}, & Z(0)=\bar{Z} \\
\dot{X}(0)=V, & \dot{Y}(0)=V, & \dot{Z}(0)=-\frac{\bar{Z}}{\Theta}
\end{array}
$$

the solution is

$X(T)=\bar{X}+V T, \quad Y(T)=\bar{Y}+V T, \quad Z(T)=\bar{Z} e^{-T / \Theta}$

where $T \geq 0$. Equations (13) are the parametric equations of a curve belonging to the plane

$X-Y+\bar{Y}-\bar{X}=0$

which is parallel to the $Z$ axis. The curve tends asymptotically to the line

$Y=X+\bar{Y}-\bar{X}, \quad Z=0$

which is also the projection of the curve in the plane $Z=0$.

\subsection{Motion of block 1 (mode 10$)$}

Since the block moves very fast, the dashpot does not take part in the motion. Therefore, the equations for $X$ and $Y$ are the same as in the elastic case, while $Z$ evolves as $X$ :

$\ddot{X}=\epsilon-(1+\alpha) X+\alpha Y$
$\ddot{Y}=0$
$\ddot{Z}=\epsilon-(1+\alpha) X+\alpha Y$.

With initial conditions

$X(0)=\bar{X}, \quad Y(0)=\bar{Y}, \quad Z(0)=\bar{Z}$

$\dot{X}(0)=\bar{V}, \quad \dot{Y}(0)=0, \quad \dot{Z}(0)=-\bar{V}$

the solution is

$$
\begin{aligned}
& X(T)=\frac{\epsilon+\alpha \bar{Y}}{\omega^{2}}+\left(\bar{X}-\frac{\epsilon+\alpha \bar{Y}}{\omega^{2}}\right) \cos \omega T+\frac{\bar{V}}{\omega} \sin \omega T \\
& Y(T)=\bar{Y} \\
& Z(T)=\bar{Z}+\bar{X}-X(T)
\end{aligned}
$$

where

$\omega=\sqrt{1+\alpha}$.

Equations (21)-(23) are the parametric equations of a line that lies on the plane $Y=\bar{Y}$ and has the equation

$Z(X)=\bar{Z}+\bar{X}-X$. 


\subsection{Motion of block 2 (mode 01$)$}

The problem is analogous to that of mode 10, but in this case $Z$ evolves as $Y$. The equations of motion are

$\ddot{X}=0$

$\ddot{Y}=\epsilon+\alpha X-(1+\alpha) Y$

$\ddot{Z}=\epsilon+\alpha X-(1+\alpha) Y$

with initial conditions

$X(0)=\bar{X}, \quad Y(0)=\bar{Y}, \quad Z(0)=\bar{Z}$

$\dot{X}(0)=0, \quad \dot{Y}(0)=\bar{V}, \quad \dot{Z}(0)=\bar{V}$

the solution is

$X(T)=\bar{X}$

$Y(T)=\frac{\epsilon+\alpha \bar{X}}{\omega^{2}}+\left(\bar{Y}-\frac{\epsilon+\alpha \bar{X}}{\omega^{2}}\right) \cos \omega T+\frac{\bar{V}}{\omega} \sin \omega T(32)$

$Z(T)=\bar{Z}-\bar{Y}+Y(T)$.

They are the parametric equations of a line that lies on the plane $X=\bar{X}$ and has the equation

$Z=\bar{Z}-\bar{Y}+Y$.

\subsection{Simultaneous block motion (mode 11)}

In this case the equations for $X$ and $Y$ are coupled, while $Z$ evolves as $Y-X$ :

$\ddot{X}=\epsilon-(1+\alpha) X+\alpha Y$

$\ddot{Y}=\epsilon+\alpha X-(1+\alpha) Y$

$\ddot{Z}+\Omega^{2} Z=0$

where

$\Omega=\sqrt{1+2 \alpha}$.

If the motion of block 1 triggers that of block 2 , initial conditions are

$X=\bar{X}, \quad Y=\bar{Y}, \quad Z=\bar{Z}$

$\dot{X}=\bar{V}, \quad \dot{Y}=0, \quad \dot{Z}=\bar{V}$

and the solution is

$$
\begin{aligned}
X(T)= & \epsilon-\frac{2 \epsilon-\bar{X}-\bar{Y}}{2} \cos T+\frac{\bar{V}}{2} \sin T+\frac{\bar{X}-\bar{Y}}{2} \cos \Omega T \\
& +\frac{\bar{V}}{2 \Omega} \sin \Omega T \\
Y(T)= & \epsilon-\frac{2 \epsilon-\bar{X}-\bar{Y}}{2} \cos T+\frac{\bar{V}}{2} \sin T-\frac{\bar{X}-\bar{Y}}{2} \cos \Omega T \\
& -\frac{\bar{V}}{2 \Omega} \sin \Omega T \\
Z(T)= & \bar{Z} \cos \Omega T+\frac{\bar{V}}{\Omega} \sin \Omega T .
\end{aligned}
$$

If the motion of block 2 triggers that of block 1 , initial conditions are

$X=\bar{X}, \quad Y=\bar{Y}, \quad Z=\bar{Z}$

$\dot{X}=0, \quad \dot{Y}=\bar{V}, \quad \dot{Z}=-\bar{V}$

and the solution is

$$
\begin{aligned}
X(T)= & \epsilon-\frac{2 \epsilon-\bar{X}-\bar{Y}}{2} \cos T+\frac{\bar{V}}{2} \sin T+\frac{\bar{X}-\bar{Y}}{2} \cos \Omega T \\
& -\frac{\bar{V}}{2 \Omega} \sin \Omega T \\
Y(T)= & \epsilon-\frac{2 \epsilon-\bar{X}-\bar{Y}}{2} \cos T+\frac{\bar{V}}{2} \sin T-\frac{\bar{X}-\bar{Y}}{2} \cos \Omega T \\
& +\frac{\bar{V}}{2 \Omega} \sin \Omega T \\
Z(T)= & \bar{Z} \cos \Omega T-\frac{\bar{V}}{\Omega} \sin \Omega T .
\end{aligned}
$$

In both cases the solution yields the parametric equations of a curve.

\section{Orbits}

We now calculate a typical orbit of the system in the phase space. We consider the projection of the orbit in the threedimensional space $X Y Z$. As initial point at $T=0$, we choose a point $P_{0}=\left(X_{0}, Y_{0}, Z_{0}\right)$ belonging to $H$, since the system is in $H$ for the most part of its lifetime. According to Eq. (14), the first segment of the orbit lies on the plane

$Y=X+p$

with

$p=Y_{0}-X_{0}$.

The orbit will intersect plane 1 or plane 2 , according to whether $Z_{0}<0$ (spring 3 is compressed) or $Z_{0}>0$ (spring 3 is stretched), respectively. The two cases are symmetric: we suppose $Z_{0}<0$.

If $P_{1}=\left(X_{1}, Y_{1}, Z_{1}\right)$ is the intersection point of the orbit with plane 1, $X_{1}$ and $Z_{1}$ must satisfy the first of Eq. (8):

$X_{0}+V T_{1}=1+\alpha Z_{0} e^{-T_{1} / \Theta}$

where $T_{1}$ is the instant of time when the representative point of the system is $P_{1}$. Solution of (51) yields

$T_{1}=\Theta W\left(\gamma_{1}\right)+\frac{1-X_{0}}{V}$

where $W$ is the Lambert function with argument

$\gamma_{1}=\frac{\alpha Z_{0}}{V \Theta} e^{-\frac{1-X_{0}}{V \Theta}}$. 
Then

$X_{1}=X_{0}+V T_{1}, \quad Y_{1}=Y_{0}+V T_{1}, \quad Z_{1}=Z_{0} e^{-T_{1} / \Theta}$.

If $\Theta \rightarrow \infty$, it results $\gamma_{1}=0$ and $W(0)=0$ : the system reduces to the case of elastic coupling, where the coordinates of $P_{1}$ depend only on the variable $p$.

The second segment of the orbit corresponds to the motion of block 1: it is obtained from Eqs. (16)-(18) with initial conditions

$X(0)=X_{1}, \quad Y(0)=Y_{1}, \quad Z(0)=Z_{1}$
$\dot{X}(0)=0 \quad \dot{Y}(0)=0, \quad \dot{Z}(0)=0$.

The solution is

$X(T)=X_{1}-\frac{U}{2}(1-\cos \omega T)$

$Y(T)=Y_{1}$

$Z(T)=Z_{1}+X_{1}-X(T)$

where

$U=2 \frac{1-\epsilon}{1+\alpha}$.

There are now two possibilities: (1) the motion of block 1 stops at a point $P_{2} \in H$; or (2) the orbit intersects line 2 while block 1 is still moving. In the former case, the system will resume mode 00 , in the latter it will enter mode 11 . We consider the former case. The coordinates of $P_{2}$ are then

$X_{2}=X_{1}-U, \quad Y_{2}=Y_{1}, \quad Z_{2}=Z_{1}+U$.

The segment $P_{1} P_{2}$ lies on the plane $Y=Y_{1}$ and belongs to the line

$Z=Z_{1}+X_{1}-X$.

After point $P_{2}$, the system enters again mode 00 and the representative point moves on the plane

$X-Y+p+U=0$.

If $P_{3}=\left(X_{3}, Y_{3}, Z_{3}\right)$ is the intersection point of the orbit with plane $2, Y_{3}$ and $Z_{3}$ must satisfy the second of Eqs. (8):

$Y_{2}+V T_{2}=1-\alpha Z_{2} e^{-T_{2} / \Theta}$

where $T_{2}$ is the time taken by the system to move from $P_{2}$ to $P_{3}$. Solution of Eq. (64) yields

$T_{2}=\Theta W\left(\gamma_{2}\right)+\frac{1-Y_{2}}{V}$

with

$\gamma_{2}=-\frac{\alpha Z_{2}}{V \Theta} e^{-\frac{1-Y_{2}}{V \Theta}}$.
Then

$X_{3}=X_{2}+V T_{2}, \quad Y_{3}=Y_{2}+V T_{2}, \quad Z_{3}=Z_{2} e^{-T_{2} / \Theta}$.

The fourth segment of the orbit corresponds to the motion of block 2: it is obtained from Eqs. (26)-(28) with initial conditions

$X(0)=X_{3}, \quad Y(0)=Y_{3}, \quad Z(0)=Z_{3}$

$\dot{X}(0)=0 \quad \dot{Y}(0)=0, \quad \dot{Z}(0)=0$.

The solution is

$X(T)=X_{3}$

$Y(T)=Y_{3}-\frac{U}{2}(1-\cos \omega T)$

$Z(T)=Z_{3}-Y_{3}+Y(T)$.

There are again two possibilities: (1) the motion of block 2 stops at a point $P_{4} \in H$; or (2) the orbit intersects line 1 while block 2 is still moving. In the former case, the system will resume mode 00 , in the latter it will enter mode 11 . We consider again the former case. The coordinates of $P_{4}$ are then

$X_{4}=X_{3}, \quad Y_{4}=Y_{3}-U, \quad Z_{4}=Z_{3}-U$.

The segment $P_{3} P_{4}$ of the orbit belongs to the plane $X=$ $X_{3}$ and is given by

$Z=Z_{3}-Y_{3}+Y$

with $Y_{4} \leq Y \leq Y_{3}$. From the coordinates of $P_{4}$ we deduce

$Y_{4}-X_{4}=p$.

Hence the system comes back to the initial plane Eq. (49). But $P_{4}$ does not belong to the segment $P_{0} P_{1}$ of the orbit, because of the value of $Z_{4}$. This contrasts with the elastic case, where all the orbits describing the alternate motion of the two blocks are periodic with period two (Dragoni and Santini, 2010). The orbit from $P_{0}$ to $P_{4}$ is shown in Fig. 3 for a choice of $P_{0}$ that would immediately give a limit cycle in the elastic case. It can be seen that points $P_{1}$ and $P_{3}$ do not fall on the dashed lines, representing the conditions for the onset of block motion in the elastic case. Explicit expressions of the coordinates of points $P_{1}$ to $P_{4}$ are given in Table 1 .

\section{Discussion}

In the case of purely elastic coupling, the long-term behaviour of the system is a limit cycle with a particular recurrence pattern of earthquakes, whichever the initial state is (Dragoni and Santini, 2010, 2011). There is an infinite number of such cycles, the shapes of which are determined by the initial position of the system in the phase space. Such a 
Table 1. Coordinates of the first singular points of an orbit with $Z_{0}<0$.

\begin{tabular}{lll}
\hline$X_{1}=V \Theta W\left(\gamma_{1}\right)+1$, & $Y_{1}=V \Theta W\left(\gamma_{1}\right)+1+p$, & $Z_{1}=\frac{V \Theta}{\alpha} W\left(\gamma_{1}\right)$ \\
$X_{2}=V \Theta W\left(\gamma_{1}\right)+1-U$, & $Y_{2}=V \Theta W\left(\gamma_{1}\right)+1+p$, & $Z_{2}=\frac{V \Theta}{\alpha} W\left(\gamma_{1}\right)+U$ \\
$X_{3}=V \Theta W\left(\gamma_{2}\right)+1-p-U$, & $Y_{3}=V \Theta W\left(\gamma_{2}\right)+1$, & $Z_{3}=-\frac{V \Theta}{\alpha} W\left(\gamma_{2}\right)$ \\
$X_{4}=V \Theta W\left(\gamma_{2}\right)+1-p-U$, & $Y_{4}=V \Theta W\left(\gamma_{2}\right)+1-U$, & $Z_{4}=-\frac{V \Theta}{\alpha} W\left(\gamma_{2}\right)-U$ \\
\hline
\end{tabular}

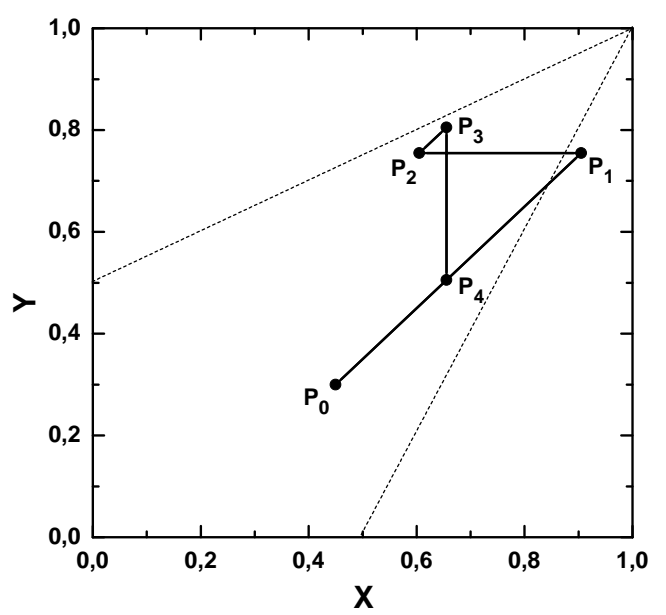

Fig. 3. Orbit with initial point $P_{0}=(0.45,0.3,-0.15)$ and with $\alpha=$ $1, \epsilon=0.7, V \Theta=1$.

position is related to the inhomogeneity of the stress applied to the fault.

If a viscoelastic coupling is present, this simple behaviour is altered. In mode 00 the forces applied to the blocks change in time as

$$
\begin{aligned}
& F_{1}(T)=-X_{0}-V T+\alpha Z_{0} e^{-T / \Theta} \\
& F_{2}(T)=-Y_{0}-V T-\alpha Z_{0} e^{-T / \Theta}
\end{aligned}
$$

There are additional nonlinear terms, with respect to the elastic case, such that the forces change non-monotonically during the loading phase of the system. Moreover the difference $F_{1}-F_{2}$ is not constant in time, implying that the stress distribution on the asperities changes during loading. This contrasts with the elastic case, where both forces are linearly increasing (in absolute value) at the same rate $V$ and their difference is constant.

As a consequence, earthquakes are anticipated or delayed with respect to the elastic case. If we consider the segment $P_{0} P_{1}$ of an orbit like the one shown in Fig. 3, the time $T_{1}$ needed for the representative point to reach plane 1 is given by Eq. (52). The corresponding time in the elastic case is

$$
T_{1}^{\prime}=\frac{1+\alpha p-X_{0}}{V} .
$$

In order to evaluate the effect of viscous relaxation, we consider the function

$$
\Delta T_{1}(X, Y, Z)=T_{1}-T_{1}^{\prime}
$$

Contour plots of $\Delta T_{1} / \Theta$ are shown in Fig. 4 on cross sections of $H$ parallel to the plane $Z=0$. If $Z_{0}<0$, such cross sections are the rectangles

$0 \leq X \leq 1+\alpha Z_{0}, \quad 0 \leq Y \leq 1$

The points where $\Delta T_{1}$ is positive represent states implying a lengthening of the interseismic time, hence a delay of the earthquake, with respect to the elastic case. The opposite holds for the points where $\Delta T_{1}$ is negative.

The importance of viscoelastic coupling depends on the product $V \Theta$ between the velocity of tectonic loading and the characteristic time of viscoelastic relaxation. For $V \Theta$ in the order of 1 or less, viscoelastic coupling is important, while it becomes negligible for larger values, say $V \Theta>10$. For a given value of $V \Theta$, larger values of $\left|Z_{0}\right|$ favour an anticipation of the earthquake, because the viscoelastic term in Eq. (76) gives an important negative contribution to $F_{1}$, hence the first condition in Eq. (7) is reached earlier.

It is also interesting to study the dependence of $T_{1}$ on the position of the initial point $P_{0}$. Contour plots of $T_{1} / \Theta$ are shown in Fig. 5 on a cross section of $H$ perpendicular to the $Y$ axis and for $Z<0$. Different values of the product $V \Theta$ are considered. The distribution of initial points with the same value of $T_{1}$ shows a remarkable change with the value of $V \Theta$. For smaller values of $V \Theta, T_{1}$ virtually depends only on $X_{0}$ and is fairly independent of $Z_{0}$ : this means that the initial stress state of the Earth's crust is irrelevant when relaxation is fast. As to the value of $T_{1}$, it may be significantly larger than $\Theta$ for smaller values of $V \Theta$.

\section{Correspondence rules}

In order to apply the model to real cases, it is necessary to assign appropriate values to the model parameters $\alpha, \epsilon, V$, 

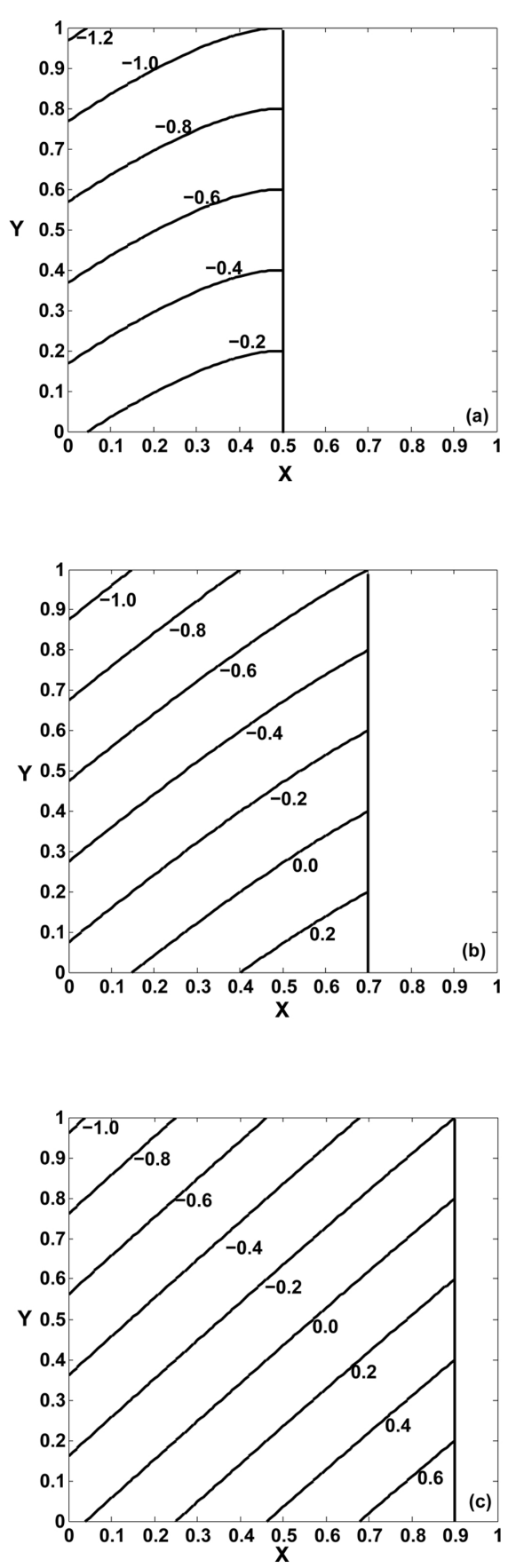

Fig. 4. Contour plots of $\Delta T_{1} / \Theta$ on different sections of $H$ : (a) $Z_{0}=$ -0.5 , (b) $Z_{0}=-0.3$, (c) $Z_{0}=-0.1(\alpha=1, V \Theta=1)$.

$\Theta$. To this aim we consider a simple model based on continuum mechanics and derive correspondence rules for the parameters of the discrete system.

We consider a plane fault embedded in a shear zone of width $d$ enclosed between rigid plates moving at relative velocity $v$. The shear zone is an elastic medium with rigidity $\mu$. The fault has two asperities with equal areas $\mathcal{A}$, located at
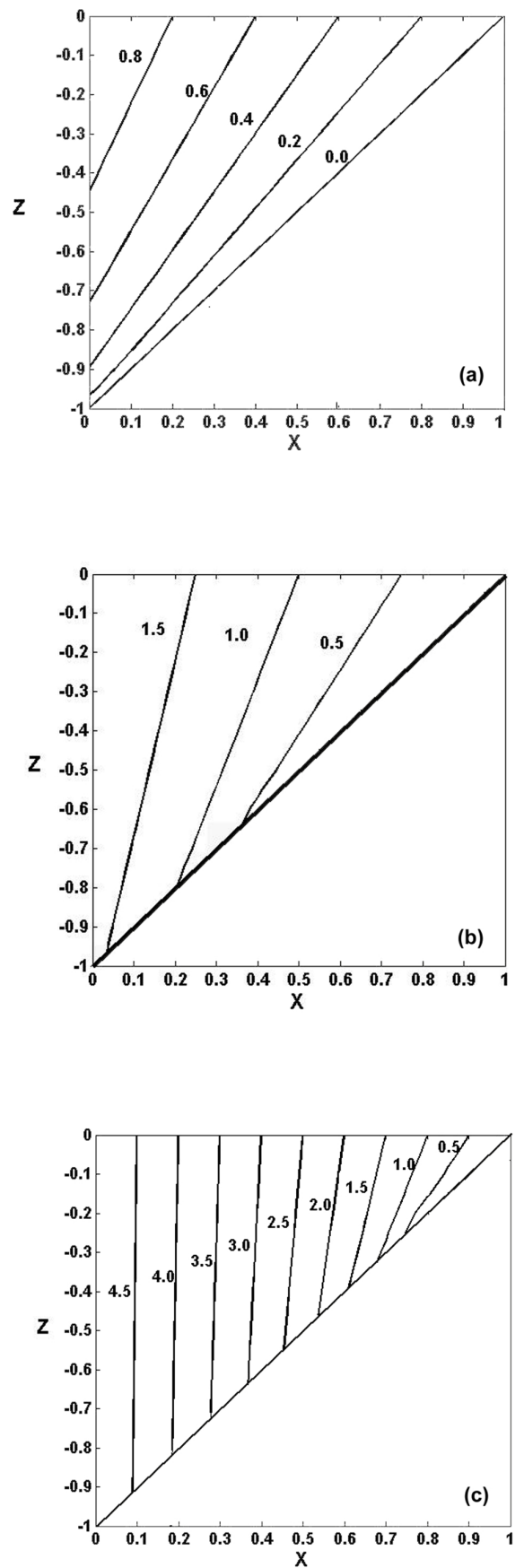

Fig. 5. Contour plots of $T_{1} / \Theta$ on a section of $H$ perpendicular to the $Y$ axis: (a) $V \Theta=1$, (b) $V \Theta=0.5$, (c) $V \Theta=0.2(\alpha=1)$.

distance $R$ from each other. The tectonic shear stress accumulating on the fault is then

$\sigma=\frac{\mu v t}{d}$

and the force on each asperity is

$f=\sigma \mathcal{A}$. 
A comparison with the force $K v t$ acting on a block in the discrete model yields the correspondence rule

$K \approx \frac{\mu \mathcal{A}}{d}$.

If we suppose that an asperity slips by an amount $u$, the shear stress produced at distance $r$ in the point-like source approximation is

$\Delta \sigma(r) \approx \frac{\mu u \mathcal{A}}{r^{3}}$.

Accordingly, the force $f$ on the other asperity is changed by an amount

$\Delta f \approx \frac{\mu u \mathcal{A}^{2}}{R^{3}}$.

In the discrete model, $u$ is the change in the difference $|x-y|$ and the change in $f$ is the quantity $K_{c} u$, whence the correspondence rules

$K_{c} \approx \frac{\mu \mathcal{A}^{2}}{R^{3}}, \quad \alpha \approx \frac{\mathcal{A} d}{R^{3}}$.

Sliding friction is a complicated function of several variables (e.g. Scholz, 1990). In compliance with the model, we make the assumption that the two asperities are characterised by a static friction $\sigma_{\mathrm{s}}$ and a dynamic friction $\sigma_{\mathrm{d}}$, so that

$\epsilon=\frac{\sigma_{\mathrm{d}}}{\sigma_{\mathrm{s}}}$.

As to $V$ and $\Theta$, they always occur as the product $V \Theta$. According to Eq. (3),

$V \Theta=\frac{K}{f_{\mathrm{s}}} v \tau$

where

$f_{\mathrm{s}}=\sigma_{\mathrm{s}} \mathcal{A}$.

Taking into account that

$u=\frac{f_{\mathrm{s}}}{K} U$

it follows

$V \Theta \approx \frac{U}{u} v \tau$.

\section{An application}

Seismic dislocations are in general complex events, with a nonuniform distribution of friction on the fault surface. In many cases, the slip distribution can be sketched as the slipping of two asperities. An example is the 1964 Alaska earthquake, one of the largest earthquakes in the last century, with

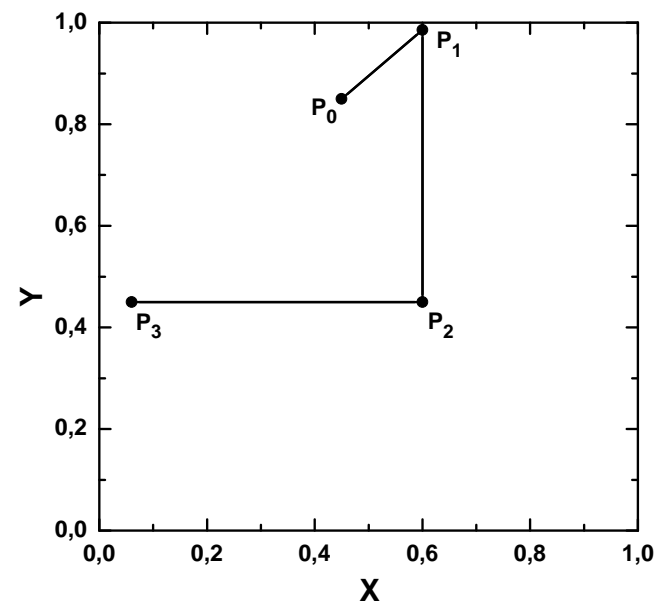

Fig. 6. Orbit with initial point $P_{0}=(0.45,0.9,0.1)$ and with $\alpha=$ $0.1, \epsilon=0.7, V \Theta=0.05$.

magnitude 9.2. Seismological, geodetic and tsunami data indicate that the earthquake was the result of the slipping of two asperities, the Kodiak Island and the Prince William Sound asperity, separated by a large spatial gap (Christensen and Beck, 1994; Holdahl and Sauber, 1994; Johnson et al., 1996; Zweck et al., 2002; Santini et al., 2003). We call them asperity 1 and 2, respectively.

Dynamical models show that the slipping of a fault made of two asperities may involve one asperity at a time or both, depending on the state of stress (Dragoni and Santini, 2010, 2011). The 1964 Alaska earthquake involved both asperities and, in particular, it was the result of the slipping of asperity 2 followed immediately by that of asperity 1 . For an application of the model, we may take $\mathcal{A} \approx 10^{4} \mathrm{~km}^{2}, R \approx 300 \mathrm{~km}$ and $d \approx 300 \mathrm{~km}$, whence $\alpha \approx 0.1$. With $\epsilon=0.7$, we have $U \simeq 0.55$.

For the Alaska earthquake, there is clear evidence of postseismic deformation occurring in the decades following the event (Zweck et al., 2002). Part of the deformation has been ascribed to aseismic slip of the fault and part to viscoelastic relaxation. The latter shows a characteristic time $\tau \approx 30 \mathrm{a}$. The relative plate velocity is $v=5.7 \mathrm{~cm} \mathrm{a}^{-1}$ (DeMets and Dixon, 1999). The average slips of the two asperities have been evaluated to about $20 \mathrm{~m}$. Then $V \Theta \simeq 0.05$, indicating a strong influence of viscoelastic relaxation on the occurrence times of earthquakes.

On the basis of the present model, we may tentatively constrain the subset of states in which the system was before 1964. In order to exhibit the observed behaviour, the initial point $P_{0}$ was such that $P_{1}$ was on plane 2 and $P_{2}$ was on plane 1 . This implies that $Z_{0}>0$ and the coordinates of $P_{0}$ satisfy the equation

$$
X_{0}+V T^{\prime}=\alpha Z_{0} e^{-T^{\prime} / \Theta}-\alpha U+1
$$




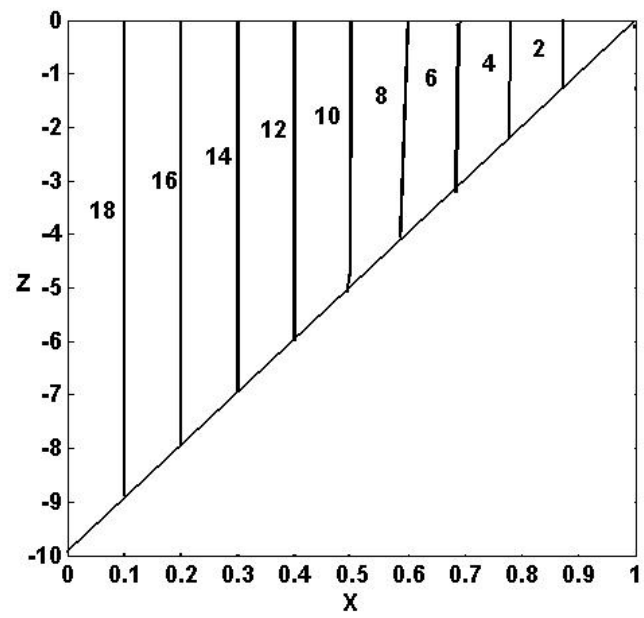

Fig. 7. Contour plot of $T_{1} / \Theta$ on a section of $H$ perpendicular to the $Y$ axis $(\alpha=0.1, V \Theta=0.05)$.

where

$T^{\prime}=\Theta W\left(\gamma^{\prime}\right)+\frac{1-Y_{0}}{V}$

with

$\gamma^{\prime}=-\frac{\alpha Z_{0}}{V \Theta} e^{-\frac{1-Y_{0}}{V \Theta}}$.

Figure 6 shows an orbit with such a property, where $P_{3}$ is a possible position of the system after the 1964 earthquake. Since $Z_{3}<0$, the next earthquake would originate from the failure of asperity 1 .

The time $T_{1}$ taken by the system to move from $P_{3}$ to $P_{4}$ can be calculated from Sect. 4 . Figure 7 shows a contour plot of $T_{1} / \Theta$ on a cross section of $H$ parallel to the plane $Y=0$, for values of the model parameters appropriate to the Alaska fault. The time $T_{1}$ can be as large as 20 times the relaxation time $\Theta$, implying an interseismic time of several centuries.

Of course this model is a strong simplification of the Alaska fault system and can only outline the broad characteristics of its seismic activity. However, it shows how discrete dynamical models can potentially seize the long-term behaviour of fault systems.

\section{Conclusions}

In many cases larger earthquakes can be considered as the effect of the failure of two or more asperities. The model presented in this paper investigates the interaction between two asperities in the presence of a viscoelastic coupling. We presented a complete analytical solution for the four modes of the system. On this basis we calculated the orbits of the system in the phase space in order to establish how the different physical quantities control the occurrence times and the source functions of the earthquakes generated by the system.
The aim of the present study is to show how a viscoelastic coupling between adjacent faults or fault segments may affect the time pattern of seismic activity. The model shows that the importance of viscoelastic coupling is controlled by the product between the velocity of tectonic loading and the characteristic time of viscoelastic relaxation. Smaller values (less than 1) of this product have a remarkable influence on the occurrence times of earthquakes originated by the twofault system.

An earthquake can be anticipated or delayed, with respect to the case of purely elastic coupling, depending on the initial state of the system, i.e. on the distribution of stress on the fault segments. Therefore, the presence of a viscoelastic coupling spoils the periodicity of seismic activity that is a property of a simple two-fault model with uniform friction: limit cycles made of alternate asperity failures are no longer produced.

We conclude that the observed aperiodicity of seismic activity can rise even in strongly symmetric models like the one considered here. An application of the model to the 1964 great Alaska earthquake suggests that viscoelastic relaxation plays a major role in controlling the occurrence times of earthquakes originated by that fault.

Acknowledgements. The authors are grateful to the editor Ilya Zaliapin and to two anonymous reviewers for useful comments and suggestions.

Edited by: I. Zaliapin

Reviewed by: two anonymous referees

\section{References}

Belardinelli, M. E. and Bonafede, M.: Post-seismic stress evolution for a strike-slip fault in the presence of a viscoelastic asthenosphere, Geophys. J. Int., 123, 744-756, 1995.

Bürgmann, R. and Dresen, G.: Rheology of the lower crust and upper mantle: evidence from rock mechanics, geodesy, and field observation, Ann. Rev. Earth Planet. Sci., 36, 531-567, 2008.

Carter, N. L.: Steady state flow of rocks, Rev. Geophys. Space Phys., 14, 301-353, 1976.

Chen, W. P. and Molnar, P.: Focal depth of intracontinental and intraplate earthquakes and their implications for the thermal and mechanical properties of the lithosphere, J. Geophys. Res., 88, 4183-4214, 1983.

Chery, J., Merkel, S., and Bouissou, S.: A Physical Basis for Time Clustering of Large Earthquakes, B. Seismol. Soc. Am., 91, 1685-1693, 2001.

Christensen, D. H. and Beck, S. L.: The rupture process and tectonic implications of the great 1964 Prince William Sound earthquake, Pure Appl. Geophys., 142, 29-53, 1994.

de Sousa Vieira, M.: Chaos in a simple spring-block system, Phys. Lett. A, 198, 407-414, 1995.

DeMets, C. and Dixon, T. H.: New kinematic models for PacificNorth America motion from $3 \mathrm{Ma}$ to present. Part 1. Evidence 
for steady motion and biases in the NUVEL1-A model, Geophys. Res. Lett. 26, 1921-1924, 1999.

di Bernardo, M., Budd, C., Champneys, A. R., and Kowalczyk, P.: Piecewise-smooth Dynamical Systems, Springer, 483 pp., 2008.

Dragoni, M. and Santini, S.: Simulation of the long-term behaviour of a fault with two asperities, Nonlin. Processes Geophys., 17, 777-784, doi:10.5194/npg-17-777-2010, 2010.

Dragoni, M. and Santini, S.: Conditions for large earthquakes in a two-asperity fault model, Nonlin. Processes Geophys., 18, 709717, doi:10.5194/npg-18-709-2011, 2011.

Dragoni, M. and Tallarico, A.: Interaction between seismic and aseismic slip along a transcurrent fault: a model for seismic sequences, Phys. Earth Planet. Inter., 72, 49-57, 1992.

Dragoni, M., Bonafede, M., and Boschi, E.: Shallow earthquakes in a viscoelastic shear zone with depth-dependent friction and rheology, Geophys. J. R. Astron. Soc., 86, 617-633, 1986.

Filippov, A. F.: Differential equations with discontinuous righthand sides, Kluwer Academic Publishers, 307 pp., 1988.

Hainzl, S., Zöller, G., and Kurths, J.: Similar power laws for foreshock and aftershock sequences in a spring-block model for earthquakes, J. Geophys. Res., 104, 7243-7253, 1999.

Harris, R. A.: Introduction to special section: Stress triggers, stress shadows, and implications for seismic hazard, J. Geophys. Res., 103, 24347-24358, 1998.

$\mathrm{He}, \mathrm{C} .:$ Interaction between two sliders in a system with rate- and state-dependent friction, Sci. China Series D, 46, 67-74, 2003.

Heki, K., Miyazaki, S., and Tsuji, H.: Silent fault slip following an intraplate thrust earthquake at the Japan Trench, Nature, 386, 595-598, 1997.

Holdahl, S. and Sauber, J.: Coseismic slip in the 1964 Prince William Sound earthquake: a new geodetic inversion, Pure Appl. Geophys., 142, 55-82, 1994.

Huang, J. and Turcotte, D. L.: Are earthquakes an example of deterministic chaos?, Geophys. Res. Lett., 17, 223-226, 1990a.

Huang, J. and Turcotte, D. L.: Evidence for chaotic fault interactions in the seismicity of the San Andreas fault and Nankai trough, Nature, 348, 234-236, 1990b.

Huang, J. and Turcotte, D. L.: Chaotic seismic faulting with massspring model and velocity-weakening friction, Pure Appl. Geophys, 138, 569-589, 1992.

Johnson, J. M., Satake, K., Holdahl, S. H., and Sauber, J.: The 1964 Prince William Sound earthquake: joint inversion of tsunami and geodetic data, J. Geophys. Res., 101, 523-532, 1996.

Kenner, S. and Segall, P: Postseismic deformation following the 1906 San Francisco earthquake, J. Geophys. Res., 105, 1319513209, 2000.

Kenner, S. and Simons, M.: Temporal clustering of major earthquakes along individual faults due to post-seismic reloading, Geophys. J. Int., 160, 179-194, 2005.

Kirby, S. H.: Rheology of the lithosphere, Rev. Geophys., 21, 14581487, 1983.

Kirby, S. H. and Kronenberg, A. K.: Rheology of the lithosphere: selected topics, Rev. Geophys., 25, 1219-1244, 1987.
Kusznir, N. J.: The distribution of stress with depth in the lithosphere: thermo-rheological and geodynamic constraints, Phil. Trans. R. Soc. Lond. A, 337, 95-110, 1991.

Lynch, J. C., Bürgmann, R., Richards, M. A., and Ferencz, R. M.: When faults communicate: Viscoelasting coupling and earthquake clustering in a simple two-fault system, Geophys. Res. Lett., 30, 1270, doi:10.1029/2002GL016765, 2003.

Marone, C., Scholz, C. H., and Bilham, R.: On the mechanics of earthquake afterslip, J. Geophys. Res., 96, 8441-8452, 1991.

McCloskey, J. and Bean, C. J.: Time and magnitude predictions in shocks due to chaotic fault interactions, Geophys. Res. Lett., 19, 119-122, 1992.

Nishimura, T. and Thatcher, W.: Rheology of the lithosphere inferred from postseismic uplift following the 1959 Hebgen Lake earthquake, J. Geophys. Res., 108, 2389, doi:10.1029/2002JB002191, 2003.

Nussbaum, J. and Ruina, A.: A two degree-of-freedom earthquake model with static/dynamic friction, Pure Appl. Geophys., 125, 629-656, 1987.

Pelletier, J. D.: Spring-block models of seismicity: review and analysis of a structurally heterogeneous model coupled to a viscous asthenosphere, in: Geocomplexity and The Physics of the Earthquakes, edited by: Rundle, J. B., Turcotte, D. L., and Klein, W., 27-42, 2000.

Piombo, A., Tallarico, A., and Dragoni, M.: Displacement, strain and stress fields due to shear and tensile dislocations in a viscoelastic half-space, Geophys. J. Int., 170, 1399-1417, 2007.

Ranalli, G.: Rheology of the Earth, 2nd Edn., Chapman \& Hall, London, 1995.

Santini, S., Dragoni, M., and Spada, G.: Asperity distribution of the 1964 great Alaska earthquake and its relation to subsequent seismicity in the region, Tectonophysics, 367, 219-233, 2003.

Scholz, C. H.: The Mechanics of Earthquakes and Faulting, Cambridge University Press, Cambridge, 1990.

Smith, B. R. and Sandwell, D. T.: A model of the earthquake cycle along the San Andreas Fault System for the past 1000 years, J. Geophys. Res, 111, B01405, doi:10.1029/2005JB003703, 2006.

Steacy, S., Gomberg, J., and Cocco, M.: Introduction to special section: Stress transfer, earthquake triggering, and timedependent seismic hazard, J. Geophys. Res., 110, B05S01, doi:10.1029/2005JB003692, 2005.

Stein, R. S.: The role of stress transfer in earthquake occurrence, Nature, 402, 605-609, 1999.

Tallarico, A., Dragoni, M., and Zito, G.: Interaction between shallow and subcrustal dislocations on a normal fault, Phys. Earth Planet. Inter., 129, 67-82, 2002.

Turcotte, D. L.: Fractals and Chaos in Geology and Geophysics, 2nd Edn., Cambridge University Press, Cambridge, 1997.

Zweck, C., Freymueller, J. T., and Cohen, S. C.: The 1964 great Alaska earthquake: present day and cumulative postseismic deformation in the western Kenai Peninsula, Phys. Earth Planet. Int, 132, 5-20, 2002. 